\title{
BEISPIELE ZYKLISCHER ELEMENTE IN MEHRSTIMMIGEN LAMENTATIONEN DES 16. UND FRÜHEN 17. JAHRHUNDERTS
}

\author{
Johannes RING
}

Hennstedt

\begin{abstract}
There are two meanings for the terminology cyclic lamentations (Lamemtationes Jeremiae Prophetae): the liturgical cyclus combinates lections - number and the textportions of the lections differ by changing time and regions. But the focus of this essay lies on the musical cyclus. Returning musical sets or particels of sets will serve to structure and to attach the lamentation.

Examples from the late 15th century show lamentations in a simple strophic form with strong orientation on cantus planus (tonus lamentationum). During the 16th century more elaborated cyclic forms had been composed - returning musical sets became paraphrased. They structure the musical cyclus while returning Jerusalem-sets have the function of a refrain.

Cyclic elements are specific to the form of the lamentations. Many composers used them in a individual manner.
\end{abstract}

\section{Zusammenfassung}

Der Begriff Lamentationszyklus (Lamemtationes Jeremiae Prophetae) hat zwei Bedeutungen: der liturgische Zyklus umfaßt je nach Zeit und Region eine bestimmte Anzahl und Kombination von Lektionen - und der musikalische Zyklus, Gegenstand der vorliegenden exemplarischen Untersuchung, in dem wiederkehrende Sätze der musikalischen Gestaltung (Strukturierung und Verklammerung) dienen.

Im späten 15. Jahrhundert lassen sich Beispiele für eine schlichte strophische, eng an den Choral (tonus lamentationum) gebundene Anlage feststellen. Im Laufe des 16. Jahrhunderts wurden differenzierte Zyklusformen entwickelt, in denen nicht nur Sätze wiederholt, sondern auch wiederkehrende Satzmodelle paraphrasiert wurden. Satzwiederholungen strukturieren Lamentationszyklen; bestimmte wiederkehrende Sätze haben Refrainfunktion.

Zyklische Elemente zeigen sich als gattungsspezifisches Gestaltungsmittel, die von vielen Komponisten individuell eingesetzt wurden.

Die folgende Schrift befaßt sich mit Vertonungen von Lamentationen des 16. und frühen 17. Jahrhunderts, die durch wiederkehrende musikalische Mittel zu Zyklen geformt werden. Im folgenden werden die fünf alttestamentarischen Bücher der Klagelieder des Propheten Jeremias, abgekürzt mit JK, mit römischen Zahlen I bis V nummeriert; arabische Zahlen geben die jeweiligen Verse an. Die Lektionen, Lectio prima, Lectio secunda und Lectio tertia, sind mit L.p., L.s. und L.t. , die Kartage, Feria quinta in coena Domini, Feria sexta in Parasceve und Sabbato sancto Paschae sind mit F.q., F.s. und S.s. abgekürzt. 


\section{Zum Begriff Lamentationszyklus}

Zunächst ist auf zwei verschiedene Bedeutungen des Begriffes "Lamentationszyklus" hinzuweisen. Der eine beschreibt ein liturgisches Ordnungsprinzip, die Vertonung einer verbindlichen Auswahl von Versen aus den Klageliedern des Propheten Jeremias, meist auf neun Lektionen verteilt. Diese liturgisch-kompositorische Einheit trifft auf eine nicht kleine Zahl von Threni-Kompositionen zu, von denen stellvertretend die Lamentationes Hieremia Prophetae ${ }^{1}$ des zeitweise in Rom wirkenden Spaniers Sebastián Raval genannt sein sollen. Ein solcher Zyklus, d.h. ein solches Ordnungsprinzip, bestehend aus 9 Lektionen, kann auch als Apparat bezeichnet werden, aus dem man die notwendigen Einzel-Lektionen auswählte. Ein solcher Lamentationszyklus wird als "kompletter Zyklus" bezeichnet. ${ }^{2}$ Besonders in der ersten Hälfte des 16. Jahrhunderts wurden entweder reine Lamentationssammlungen oder, nicht nur für die Karwoche bestimmte Sammlungen mit verschiedenen liturgischen Stücken, bei denen einige Gattungen mehrfach vertreten sind, angelegt.

Das Repertoire der Lamentationen innerhalb der einzelnen Sammlungen divergiert stark, "denn bekanntlich waren in der römischen Kirche die Lesungen der Karwochenmatutinen bis zur Mitte des 16. Jahrhunderts ... noch nicht einheitlich festgelegt, sondern richteten sich nach örtlichen Gewohnheiten, die zum Teil beträchtliche Unterschiede aufwiesen."3 Aus diesen Lamentationssammlungen konnten entsprechende Lamentationssätze oder Zyklen ausgewählt werden. Als Beispiel für einen reinen Sammelpublikation sei hier Lamentationum Jeremie prophete liber primus, 1506 von Petrucci in Venedig gedruckt, genannt. Ein anderes Beispiel ist die Handschrift I-Rvat C.G. XII. aus dem Jahr 1543, die verschiedene Lamentationen z.B. des in der päpstlichen Kapelle in Rom wirkenden Spaniers Juan Escribano enthält. Der Schreiber von 1543 betitelt Escribanos Zyklus zu 4 bis 6 Stimmen mit super Lamentationes una cum Oratio Hieremie Prophete. Die ausdrückliche Erwähnung der Oratio, die einen liturgischen Lamentationszyklus abschließt, kann für die Benutzer des Chorbuches als Hinweis auf einen Lamentationszyklus aufgefaßt werden. Doch umfaßt Escribanos Zyklus tatsächlich nur sechs Lektionen, was zu verschiedenen Spekulationen zur Liturgie oder den Aufführungsmodalitäten führt.

Drucktitel mehrstimmiger Lamentationen aus dem 16. Jahrhundert weisen auf zyklische Intentionen der Publikationen hin. ${ }^{4}$ Drucküberschriften wie Officium (Hebdomadae Sanctae), z.B. bei Giammateo Asola (1584), ${ }^{5}$ zeigen offensichtlich liturgisch vollständige Offizienzyklen

1. Romce / Apud Nicolaum Mutium / 1594. Neben einem liturgisch vollständigen Zyklus mit neun Lektionen enthält Ravals Druck den Canticum Zachariae "Benedictus Dominus Deus", den 50. Psalm und eine Huldigungsmotette mit einem weihnachtlichen Text. Ravals Lamentationen sind Gegenstand weiterer Forschungen des Autors dieses Aufsatzes.

2. Vergl. Magda Marx-Weber, (Art.) Die mehrstimmigen Lamentationen des 15. und 16. Jahrhunderts, in: Die Musik in Geschichte und Gegenwart, zweite neubearbeitete Ausgabe, hrsg. von Ludwig Finscher, Sachteil 5, Kassel u.a., 1996 [= Marx-Weber, 1996], Sp. 897.

3. Günter Massenkeil, Mehrstimmige Lamentationen aus der ersten Häfte des 16. Jahrhunderts, Mainz 1965. S. 6*. [= Massenkeil, 1965]

4. Der Autor konnte, gestützt auf die relevanten Artikel in The New Grove, London 1980, MGG, Kassel 1940f. und Angaben in RISM bisher ungefähr 100 Überschriften und Titel mehrstimmiger Musik für die Karwoche überprüfen.

5. Zur Auflistung der Drucktitel Asolas siehe: The New Grove, Bd. 1, London 1980, S. 657. 
an, in denen neben Responsorien und/oder Passionen auch die Lamentationen vertont sind. Eine andere Auffassung von Vollständigkeit ergibt sich aus Überschriftpassagen wie maiori hebdomada, in triduo hebdomadae sanctae und wenn alle drei Kartage vor Ostern einzeln aufgezählt sind. ${ }^{6}$ In solchen Sammlungen liegen Lektionen für alle drei Kartage vor, doch müssen damit nicht drei Lektionen für jeden Kartag gemeint sein, wie man bei den handschriftlich überlieferten Lamentationen des Portugiesen Estévão De Brito ${ }^{7}$ erfahren kann. Der Drucktitel Liber Lamentationum, wie bei Elzéar Carpentras (1532) ${ }^{8}$ steht offensichtlich für ein Buch, das ausschließlich Lamentationen enthält, die durch ihren Umfang, besser: ihre liturgische Vollständigkeit, in Carpentras Fall geordnet zu $3 \times 3$ Lektionen, die Publikation als eigenständiges Musikbuch rechtfertigen.

Andere Lamentationen erscheinen nicht in Zyklen für die Karwoche, sondern sind Bestandteile großer Kollektionen bzw. Publikationen für das ganze Kirchenjahr. So sind Jacob Handls (Gallus) Lamentationen im 3. Teil seines Opus Musicum von 1587, ein vier Bücher umfassender Proprium-Druck, zu finden. ${ }^{9}$

Im Zusamenhang mit norditalienischen Lamentationen ist von zyklischer Vereinheitlichung und zyklischen Elementen die Rede. ${ }^{10}$ Hier wird der Zyklus-Begriff in seiner musikalischen Bedeutung verwendet: in den Lamentationen werden Kompositionsmittel eingesetzt, die eine Folge von Lektionen musikalisch miteinander verzahnen. Solche musikalischen Lamentationszyklen entsprechen nicht automatisch einer liturgisch vollständigen Textauswahl, wie sie liturgische Formulare vorgeben. Verschiedene Beispiele sollen nun die musikalische Zyklusgestaltung vorstellen.

\section{Kompositorische Verzahnung in Einzel-Lektionen}

Ein anonymer Komponist (Anonymus I), ${ }^{11}$ dessen Lamentation im Lamentationum Jeremie prophete liber primus 1506 von Petrucci in Venedig gedruckt erschien, mag als Beispiel dienen, wie ein einzelner Lamentationssatz zyklisch gestaltet werden kann. So benutzt er ein gleichbleibendes Vokalisenmodell als Gestaltungsmittel, um seiner dreistimmigen Lektion Geschlossenheit zu geben. Der Einsilbigkeit des Beth, JK. I. 2, trägt der unbekannte Komponist durch Auslassung des Auftaktes Rechnung. Durch Paraphrasieren verquickt er den Jerusalemrefrain mit dem vorherigen Vers.

6. Z.B. bei A. Bonavita (1600): Hieremiae lamentationes magnam in omnium pietatem, \& religionem in deum excitantes: tribus sacro sanctae hebdomadae diebus..., Venedig, 1600. RISM B4947.

7. Estévão De Brito, Vol. II. Obras Diversas, Portugaliae Musica, hrsg. von Miguel Querol Gavaldà, Lisabon 1976, S. XI und 54-67.

8. Elziarii Geneti (Carpentras), Opera Omnia, CMM 58, II, hrsg. von Albertus Seay, American Institute Of Musicology, 1973. S. XI [Carpentras, CMM, 1973.]

9. Jacob Handl (Gallus), Opus Musicum III, verl. von E. Bezecny und J. Mantuani, in: DTÖ, Jg. XV/1 - Band 30, Repr., Graz 1959.

10. Marx-Weber, 1996. Sp. 899.

11. Massenkeil, 1965, S. 14-18. Massenkeil ediert außerdem in dieser Ausgabe folgende Lamentationen, die in dieser Schrift aufgeführt sind: Anon. II, S. 112-119, Anon. IV, S. 142-148, Johannes De Quadris, S. 24-32, Antoine Fevin, S. 103-111, Johannes Gardano, S. 60-79, Erasmus Lapicida, S. 51-59, Claudin de Sermisy, S. 120-126 und Bartolomeo Tromboncino, S. 33-50. 
Notenbeispiel 1 (Anonymus, "Migravit Judas", JK. I. 3 und “Jerusalem")
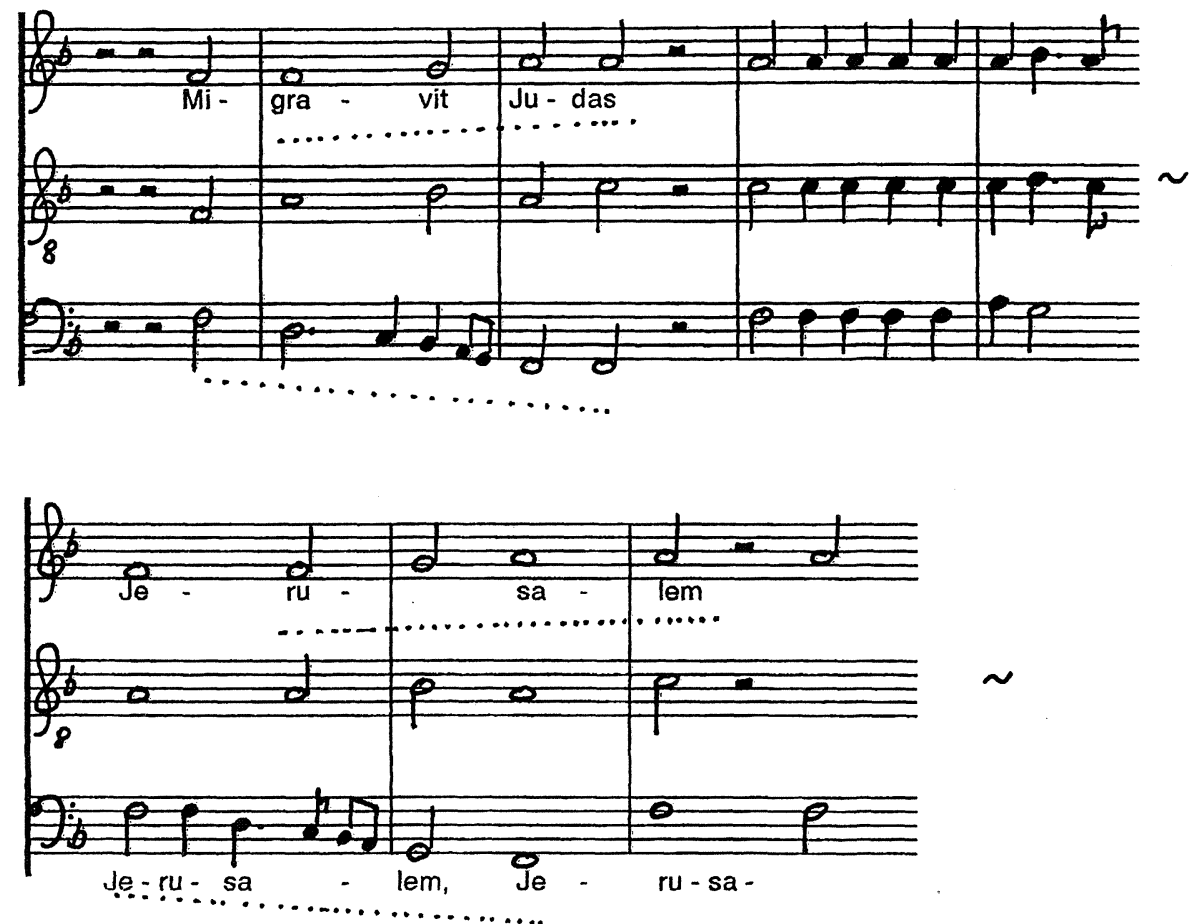

Identische Buchstaben-Sätze sind nicht nur Gestaltungsmittel, um Einzel-Lektionen musikalische Geschlossenheit zu geben, sie dienen auch der musikalischen Geschlossenheit in Lamentationszyklen.

\section{Zum Aufbau von Lamentationszyklen}

Am Anfang der Geschichte der mehrstimmigen Lamentationen steht Johannes de Quadris Zyklus, der in der Handschrift I-VIs U VIII 11 aus den Jahren 1430-40 überliefert ist. "Die zweistimmigen Lektionen sind konsequent strophisch gebaut: nicht nur die Buchstabensätze sind untereinander gleich, auch die Verse sind, mit kleinen Variationen, über ein einziges Modell gearbeitet." ${ }^{12}$ Der strophische Aufbau zeigt sich als prägendes Merkmal, was mit der starken Bindung an den Choral, respektive den Lektionston erklärt werden kann. Der strophische Aufbau bei de Quadris kann als das einfachste Mittel der zyklischen Gestaltung von Lamentationen bewertet werden.

12. Marx-Weber, 1996, Sp. 898. 
Ein halbes Jahrhundert später findet sich bei Alexander Agricola ${ }^{13}$ zyklische Geschlossenheit durch wiederkehrende Jerusalemrefrains (Jerusalem ut supra). Dieses zyklische Gestaltungsmittel findet sich auch in Agricolas vierstimmigen Lamentationszyklus. Eine Generation später wendet Stephan Mahu ein Kombinationsverfahren bei den Jerusalemrefrains an, ${ }^{14}$ nämlich "daß auf jeden der drei Tage die erste Lection mit ein und demselben Jerusalem geschlossen wird; daß die zweite Lection auf Feria quinta und Feria sexta, und endlich wiederum die dritte Lection auf Feria quinta und Feria sexta dieselbe gleiche Komposition zum Jerusalem als Schluß enthalten." 15

Mit Antoine Fevins vierstimmigen Lamentationen ${ }^{16}$ liegt ein weiterer Zyklus vor, in dem der gleiche Jerusalemrefrain die drei Lektionen beschließt. Entsprechend der textlichen Vorlage des Officiums ${ }^{17}$ wird das Wort Jerusalem zweimal vertont, sonst bringt der knappe Lamentationszyklus in den Vers-Sätzen und dem Refrain keine Wortwiederholungen.

In andere Lamentationen ist festzustellen, daß die Idee der wiederkehrenden Jerusalemrefrains keineswegs so systematisch durchgehalten wird. So beschließt ein anderer anonymer Lamentationszyklus (Anonymus II) die ersten beiden Lektionen mit ein und demselben Jerusalemrefrain. Die dritte Lektion versieht der anonyme Komponist, dessen Zyklus, wie der von Fevin, 1549 von Montanus-Neuber gedruckt wurde, mit einem neuen Refrain, der sich besonders klanglich durch seine tiefe Disposition ${ }^{18}$ von den anderen unterscheidet. Außerdem wechselt der (variierte) C.f. in die zweite Stimme. Eine Paralelle zeigt der Lamentationszyklus von Claudin De Sermisy aus dem gleichen Nürnberger Druck, ${ }^{19}$ in dem die ersten beiden Lektionen mit einem identischen Jerusalemrefrain beschlossen werden. Die dritte Lektion wird mit einem neuen Refrain beendet, der sich stilistisch durch Dominanz längerer Notenwerte von dem ersten Refrain-Modell unterscheidet.

Eine andere Spielart der Vokalisen-Wiederholung bringen Bartolomeo Tromboncinos Lamentationen. Der Aleph-Satz am Anfang der 1. Lektion (JK. I. 1) ist identisch mit dem ZainSatz (JK. I. 7) in der 2. Lektion, mit dem Teth-Satz (JK. I. 9) und dem Lamed-Satz (JK. I. 12) in der 3. Lektion und dem Mem-Satz (JK. I. 13) in der letzten Lektion. In der ersten und letzten Lektion folgt dieser Satz direkt auf die Exordium-Sätze "Incipit Lamentatio" und "Sequitur de Lamentatione", in der 2. Lektion leitet er den 3. Vers und in der 3. Lektion den 2. und 5. Vers ein. Ein anderer Vokalisensatz mit Variante scheint Verwandtschaft mit dem erstbesprochenen zu haben.

13. Alexander Agricola, Opera Omnia, CMM 22, Vol. III, hrsg. von Edward R. Lerner, American Institut Of Musicology, 1966.

14. In: Selectae harmoniae..., gedruckt von Georg Rhaw, Wittemberg 1538.

15. Carl Dreher, Die Lamentationen des Stephan Mahu, Monatshefte für Musik-Geschichte, VI. Jg. (1874/4). S. 58.

16. Zwei der Lektionen werden auch seinem Bruder Robert de Fevin zugeschrieben; vergl. Howard Mayer Browns Artikel Antoine de Fevin und Robert de Fevin, in: The New Grove, Bd. 6, London 1980. S. 516/17.

17. Vergl. Liber Usualis Missae Et Officii, Paris u.a. 1964, S. 631-762.

18. Die obere Stimme wechselt von Diskant- in den Altschlüssel und die anderen Stimmen halten sich auch in der tiefsten Lage ihres Registers auf.

19. Claudin De Sermisy, Opera Omnia, CMM 52, II, hrsg. von Gaston Allaire, American Institute Of Musicology, 1972. S. 1ff. und Massenkeil, 1965. 
Notenbeispiel 2 (1. Vokalise (z.B. Aleph),2. Vokalise (z.B. Daleth) und 3. Variante bei Beth)
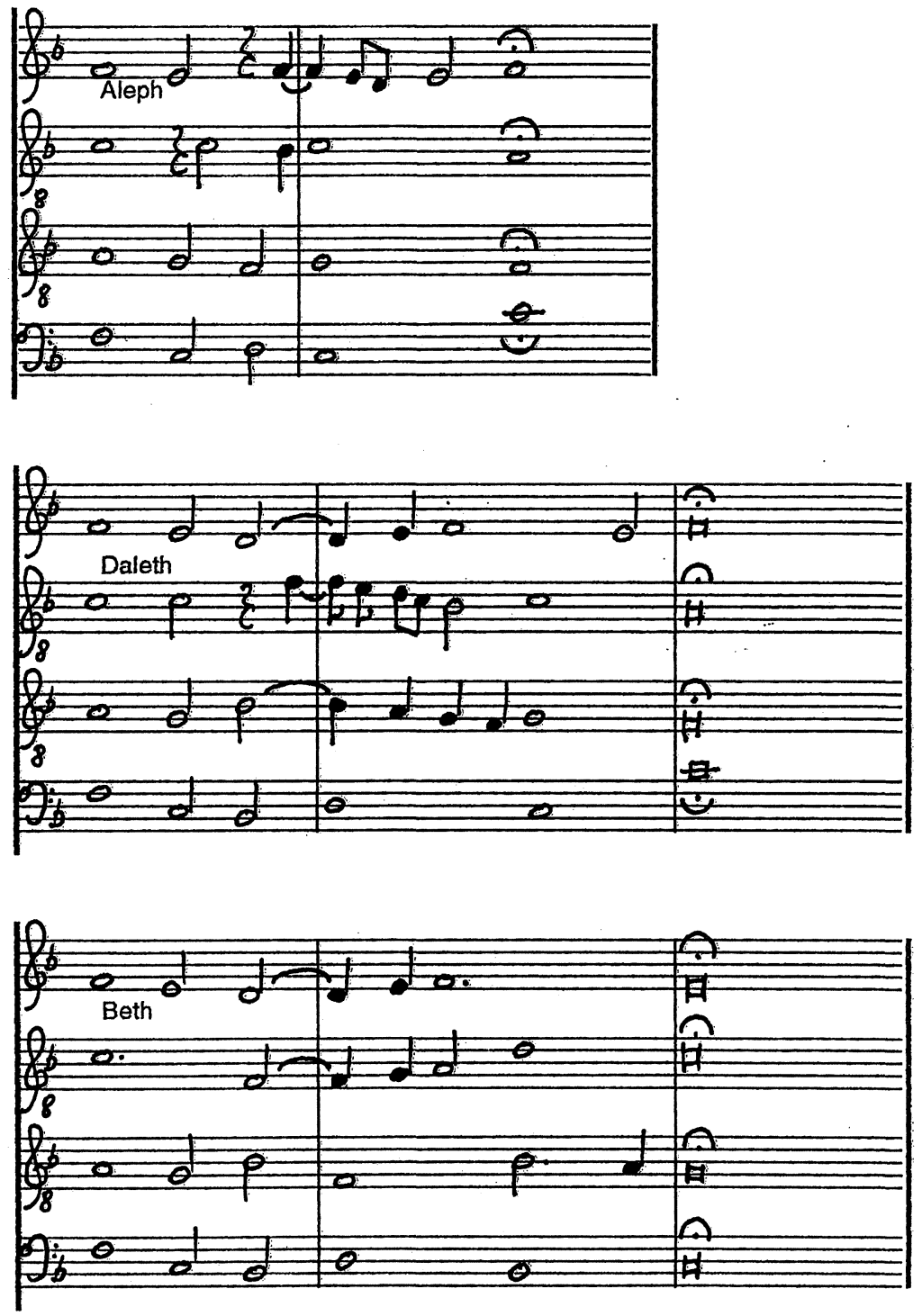

Die 2. Vokalise kommt viermal vor, die Variante zweimal, einmal in der ersten und der letzten Lektion. 
In Tromboncinos Lamentationszyklus gibt es eine einmalige Vokalise, die der Komponist als Modulations-Brücke einsetzt, um in den 6. Modus zurückzugelangen. Dieser Vau-Satz verbindet in der 2. Lektion den ersten und zweiten Vers (JK. I. 5 \& 6). Überhaupt zeigt dieser VersSatz eine große harmonische Vielfalt, z.B. in Form von Querständen.

Noch ein weiterer Buchstaben-Satz tritt dreimal auf. Er kann, wie das Notenbeispiel zeigt, als rudimentäre Klauselkombination, als absolute Reduktion der ersten Vokalise angesehen werden.

Notenbeispiel 3 (Ghimel- und Alephsatz)
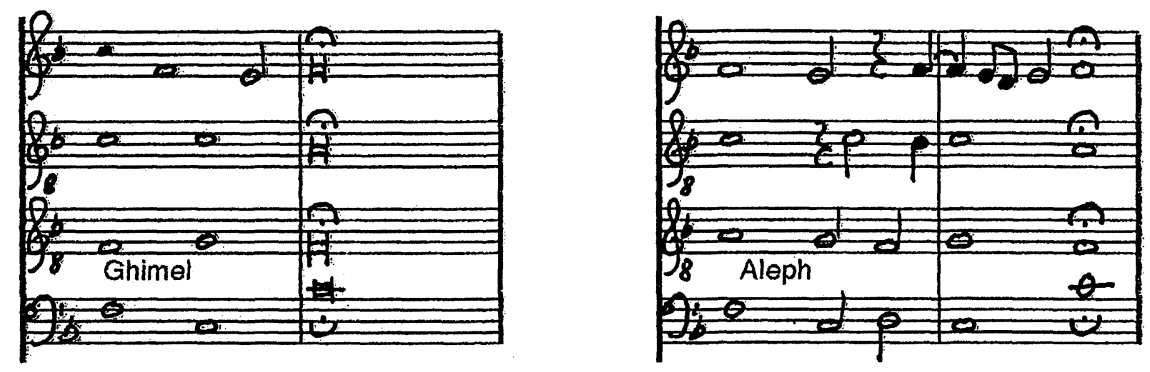

Tromboncino stellt durch wiederkehrende, gut erkennbare Vokalisen musikalische Zusammenhänge her, ohne, wie der anonyme Komponist der florentinischen Handschrift, in eine trokkene Schematik zu verfallen. Zu erwähnen ist noch, daß in Vers-Passagen ähnliche und identische Wendungen aus den Vokalisen (oder vice versa) feststellbar sind, eine Art kompositorische Bausteintechnik, die sich als Tromboncinos Kompositionsstil in den Lamentationen erkennen läßt.

Im gleichen Druck mit Lamentationen von 1509 veröffentlichte Petrucci einen Lamentationszyklus von Erasmus Lapicida, dessen variierende Behandlung der Vokalisen Ähnlichkeiten mit Tromboncini zeigt, wobei die Wiederkehr eines Modells bei Lapicida noch unregelmäßiger erscheint. Lapicidas Ghimel- und Daleth-Satz am Ende der 1. Lektion (JK. I. 3) und am Anfang der 2.Lektion (JK. I. 4) gleichen sich sehr. Denkbar ist, daß der Komponist mit diesem Vokalisenmodell beide Lektionen musikalisch verbinden wollte.

Ein weitere Vokalise kehrt, wie die zuerst aufgeführte, wieder. Es handelt sich um den Beth-Satz der 1. Lektion und den He-Satz aus der 2. Lektion. Auch diese beiden Vokalisen mögen von Lapicida als musikalische Verzahnung zweier Lektionen komponiert worden sein. Die zyklische Verzahnung der 2. und 3. Lektion erfolgt bei Lapicida nicht über wiederkehrende Vokalisenmodelle, sondern über identische Jerusalem-Refrains.

In Gardanos Lamentationen, ebenfalls im Nürnberger Druck von 1549, wird ein Vokalisenmodell variiert. Das imitatorisch aufgebaute Aleph, mit dem die Lamentationen beginnen, wird in einen kompakten kurzen Satz (Buchstabe He) verwandelt. Das Ghimel (JK. II. 3) der 2. 
Lektion und das He (JK. II. 5) stellen. weitere Paraphrasen dar. Auch das zweistimmige Beth (JK. II. 2) kann auf das anfängliche Modell bezogen werden. ${ }^{20}$

Notenbeispiel 4 (Gardano; Aleph-Modell und "Derivate")
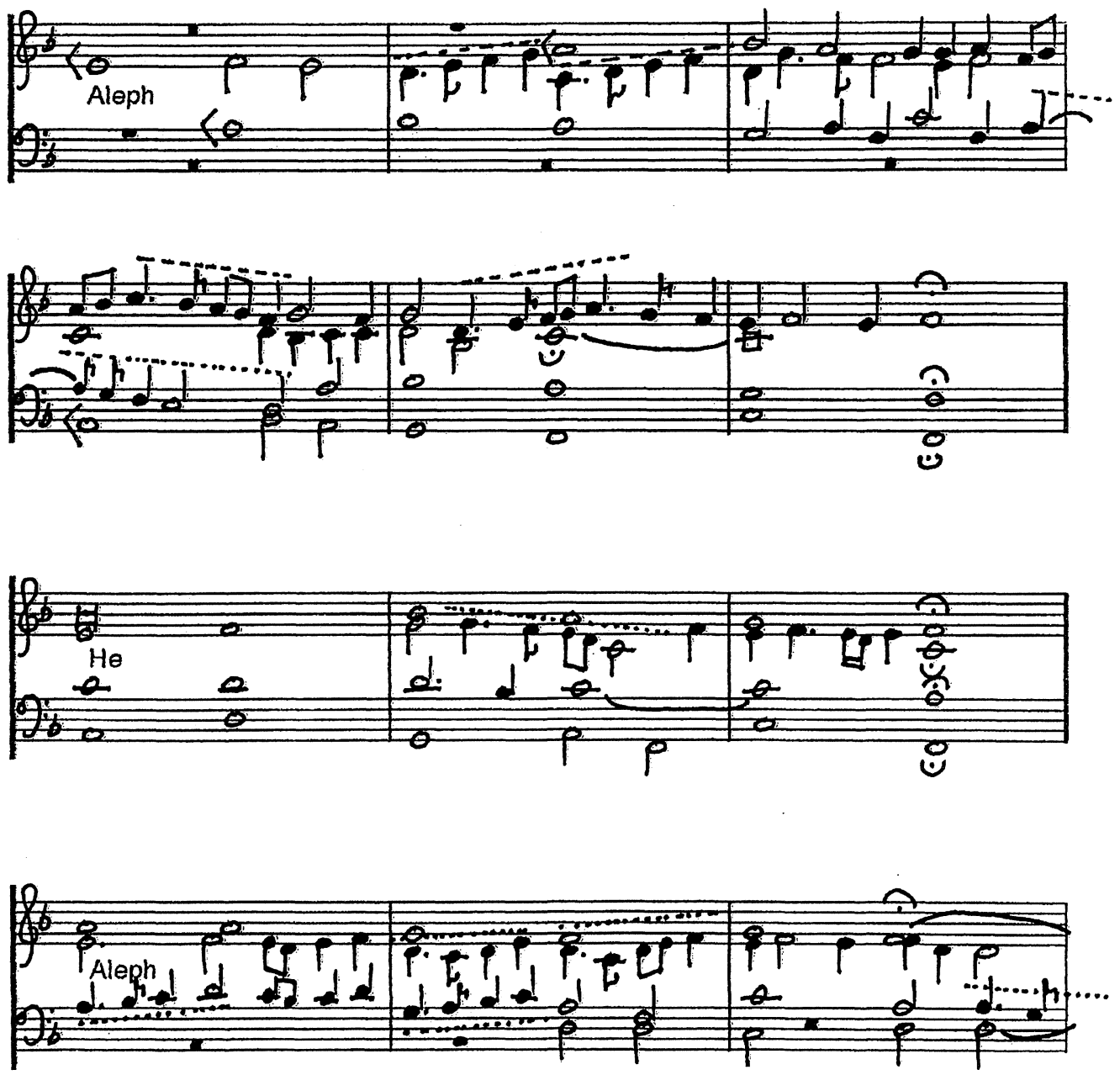

20. Möglicherweise wollte der Komponist mit der Zweistimmigkeit des Beth-Satzes und des halben Vers-Satzes die numerische Position des Threni-Verses illustrieren. 

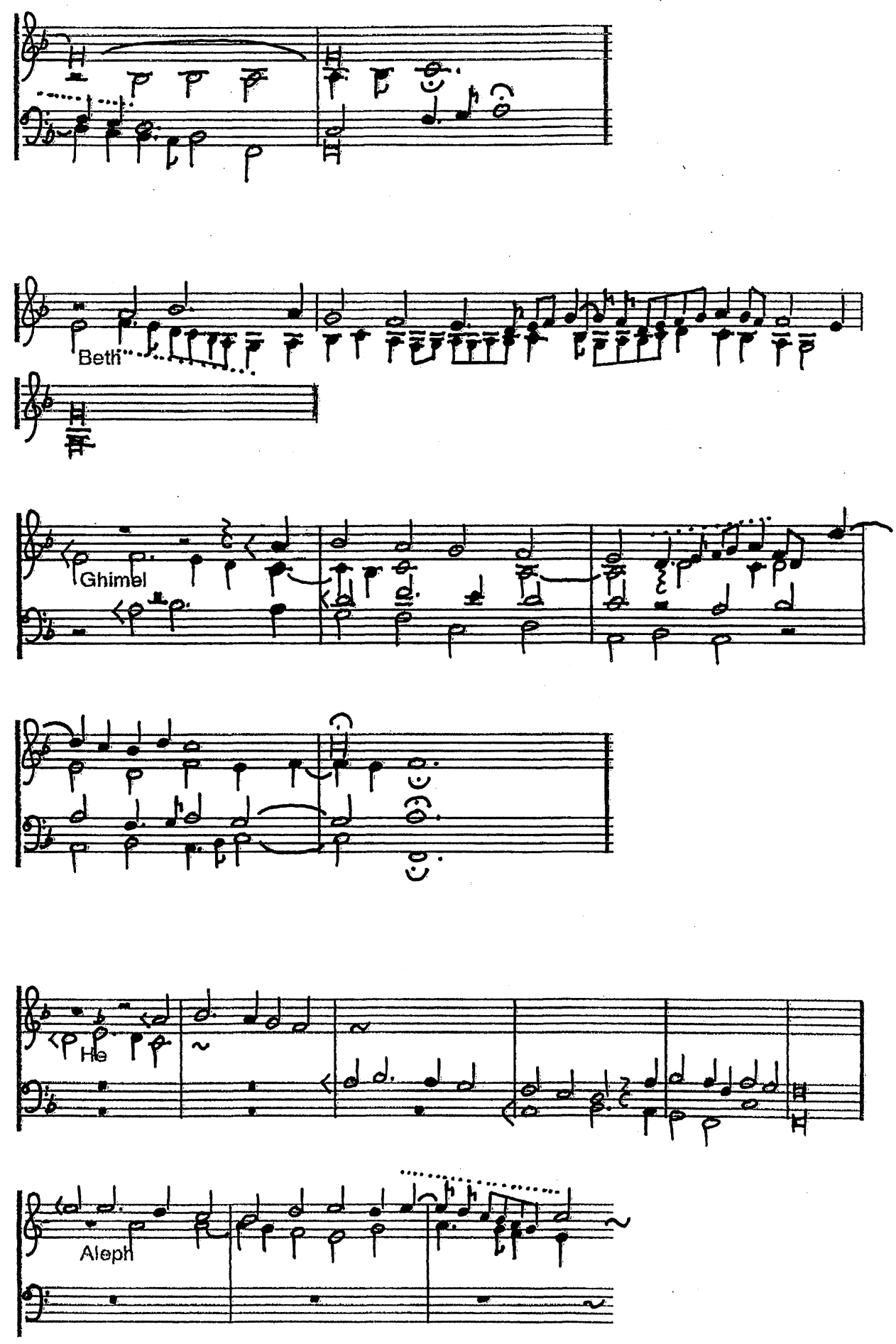
Die Tricinien Aleph und Beth (JK. IV. 1 \& 2) erinnern in ihrem tonalen und imitatorischen Anfang an das gleiche Urmodell, stehen aber für einen Moduswechsel, der die $a-e$-Quintlage zu der Finalis $c$, Quintlage $c-g$, führt. Zwischen dem Beth-Satz und dem folgenden Vers kommt es zu keiner Zäsur. Das Prinzip der deutlichen Trennung von Vokalisen- und Vers-Satz scheint hier für einen Moment mittels einer Cadenza fugite aufgehoben. Der Ghimel-Satz dieser 3. Lektion (JK. IV. 3) läßt sich als entfernt verwandt mit dem ersten Buchstaben-Satz auffassen. Der Daleth-Satz scheint sich als Paraphrase wieder mehr bei der Ur-Vokalise Aleph anzulehnen. Besonders eindrucksvoll wirkt die absteigende Fanfare der unteren Stimme. In den letzten beiden Versen der Lektion steigert sich die Besetzung zur Fünfstimmigkeit. Die beiden dazugehörigen Vokalisen lassen sich auf das dreistimmige Modell vom Anfang der Lektion zurückführen.

Bemerkenswert ist auch, wie die aufsteigende Floskel von Minimae, siehe Aleph-Satz, im Verlauf der folgenden Vokalisen-Sätze, aber auch in den Vers-Sätzen eine Steigerung erfährt. Im Vau-Satz des letzten Verses (JK. IV. 6) wird sie zum prägenden Bestandteil.

Johannes Gardano ist der Komponist eines Lamentationszyklus, in dem die drei Lektionen durch Satz-Modell-Paraphrasen miteinander verzahnt sind. Anders als bei wiederkehrenden identischen Buchstaben-Versen, wie beispielsweise in der einzelnen Lamentation des Anonymus I, identischen Jerusalemrefrains wie bei Fevin oder der Kombination gleicher Vokalisen- und Jerusalemsätze wie bei De Quadris, zitiert Gardano kaum wörtlich einen Satz. Sein kompositorisches Anliegen liegt in der Satzvariation, die er souverän handhabt; diese Variationstechnik führt, anders als bei den drei genannten Komponisten, zu einem indirekten Zusammenhalt der Lektionen. Satzverwandtschaften und Abwechslungsreichtum bis hin zum Kontrast des Moduswechsels kennzeichnen Gardanos Lamentationszyklus, dessen äußerliche Auffälligkeit das Fehlen von Jerusalemrefrains ist. Diese mögen choraliter ausgeführt worden sein, was zum einen einen Kontrast zur sonstigen Mehrstimmigkeit darstellt, zum anderen aber auch dem zyklischen Gestaltungsprinzip dienlich ist, kehrt doch der chorale Jerusalem-Vers immer unverändert wieder. Möglicherweise wurde in der Praxis hier ein Jerusalem-Satz im gleichen Modus aus einer fremden Komposition angefügt. Einige Komponisten, wie z.B. E. Carpentras ${ }^{21}$ verfaßten Alternativsätze, die zum Austausch im eigenen Zyklus bestimmt waren. Auf der anderen Seite zeigt das Manuskript der Cavalieri-Lamentationen, daß unter den "twelve substitute choral verses and letters" 22 tatsächlich auch fremde Sätze, in diesem Fall ein siebenstimmiger Jerusalemsatz von Costanzo Festa, Verwendung fanden.

In seinem Lamentationszyklus stellt sich Johannes Gardano, über den keine biographischen Fakten greifbar sind, als bedeutender Komponist der ersten Hälfte des 16. Jahrhunderts vor.

Ein kleiner anonymer Lamentationszyklus in einer florentinischen Handschrift (Anonymus IV) vom Beginn des 16. Jahrhunderts, kombiniert systematisch zwei zyklische Gestaltungsmerkmale:

21. Carpentras, CMM, 1973.

22. Vergl. Emilio De'Cavalieri, The Lamentations And Responsories Of 1599 And 1600, in: Miscellanea 5, Early Sacred Monody, hrsg. von Murray C. Bradshaw, American Institute Of Musicology, Hännsler-Verlag, 1990, s. XIV. [Cavalieri] 
zum einen bringen identische Jerusalem-Sätze - einige Durchgänge lockern die Stimmverläufe auf - zum anderen das gleichbleibende Satzgerüst der Vokalisen musikalische Geschlossenheit. ${ }^{23}$

Ein weiteres systematisch in norditalienischen Lamentationen eingesetztes Kompositionsmittel beschreibt John Bettley. ${ }^{24}$ In den 1580er Jahren gingen einige norditalienische Komponisten dazu über, Vers-Sätze ihrer Lamentationen in Falsobordone zu setzen. Als Beispiel nennt Bettley Pietro Vincis Lamentationen (1583), ${ }^{25}$ in denen nicht nur Vers-Passagen, sondern auch die Buchstabensätze im unveränderten Falsobordone-Modell wiederkehren. Als weiteres Beispiel sind hier Orazio Vecchis Lamentationen (1587) zu nennen, "in which the three verses for each lectio share the same shematic falsobordone material ..."26 In diesem Lamentationszyklus sind die Vokalisen und Jerusalem-Refrains in mensurierter Polyphonie komponiert.

Auch wiederkehrende Jerusalemrefrains können norditalienischen Komponisten als beliebtes zyklisches Gestaltungsmittel ihrer Lamentationen attestiert werden. Diese Gepflogenheit hat ihren Ausgang am Anfang des Jahrhunderts - als Beispiel wurde schon der Lamentationszyklus von Tromboncini (s.o.) genannt. Bettley führt 25 norditalienische Lamentationen auf, in denen Jerusalem-Sätze wiederholt werden. ${ }^{27}$

Mit Orlando di Lassos vierstimmigen Lamentationen liegt ein weiterer Zyklus aus der zweiten Hälfte des 16. Jahrhunderts vor, in dem Sätze wiederkehren. ${ }^{28}$ Anders als in den Lektionen 4 bis 9 behält der Komponist in der L.p., L.s. und L.t. des Primi Diei den Modus bei, was ihm ermöglicht, diese drei Lektionen mit dem gleichen Jerusalem-Satz zu beschließen. "Damit wirkt es wie ein Refrain am Ende jeder Lektion. Der wiederholt auftretende C.f. bei den hebräischen Buchstaben in I, 1-3 ist ebenso ein weiterer, wenn auch weniger wörtlicher Refrain. Die drei Lektionen sind somit musikalisch in hohem Maße vereinheitlicht." ${ }^{29}$ Dieses vereinheitlichende Verfahren wird in den folgenden Lektionen modifiziert: " In II, 3 und III, 1, wo jede der dreifachen Wiederholungen der hebräischen Buchstaben mehrstimmig vertont sind, wird jeweils der ganze Satz bei jedem Block der drei Buchstaben wörtlich wiederholt: In II, 3 z.B. sind die drei "Aleph" untereinander identisch, ebenso die drei "Beth" und so fort." 30

Im fünfstimmigen Zyklus von 1585, der fast zeitgleich mit Lamentationszyklen von T.L. da Victoria und G.P. da Palestrina im Druck erschien, wiederholt Lasso weder Buchstaben- noch Jerusalemsätze, womit er im Aufbau römischen Gepflogenheiten entspricht. ${ }^{31}$

Mit Jacob Handls (Gallus) Lamentationen, die er im 3. Teil seines Opus Musicum 1587 veröffentlichte, ${ }^{32}$ liegt ein weiterer Zyklus mit wiederkehrenden Buchstaben-Sätzen und Jerusa-

23. Ausnahme ist das aus dem Rahmen fallende Beth zu Beginn des JK. I. 2

24. La compositione lacrimosa: Musical Style and Text Selection in North-Italian Lamentations Settings in the Second Half of the Sixteenth Century, JRMA, 118, 2 (1993). 167-202. [= Bettley, 1993].

25. Vergl. ebda. S. 181.

26. Ebda., S. 182.

27. Vergl. ders. S. 184.

1992].

28. Orlando di Lasso, Lamentationes Jeremiae Prophetae, hrag. von Peter Bergquist, Kassel u.a., 1992. [Lasso,

29. Lasso, 1992. XI.

30. Ebda.

31. Vergl. Lasso, 1992. IX.

32. Siehe Anmerkung 9. 
lem-Refrains vor. Die Aleph-, Beth- und Gimel-Sätze der L.p. sind identisch mit den Vau-, Daleth- und He-Sätzen der L.s.. Beide Lektionen schließen mit dem gleichen Jerusalem-Satz.

Die identischen Buchstaben-Sätze treten folgendermaßen auf: der erste Buchstaben-Satz der L.p. entspricht dem letzten Buchstaben-Satz der L.s., der zweite Buchstaben-Satz der L.p. entspricht dem ersten Buchstaben-Satz der L.s., der dritte Buchstabe der L.p. entspricht dem zweiten Buchstaben-Satz der L.s.. Handl setzt mit gleichen Buchstabensätzen am Anfang der ersten und am Ende der zweiten Lamentation eine musikalische Klammer. Mit den anderen wiederkehrenden Sätzen an unterschiedlichen Positionen verzahnt er beide Lamentationen, eine Verzahnung, die an die über 80 Jahre früher publizierten Lamentationen von Lapicida (s.o.) erinnert.

Ähnlich verzahnt Handl die dritte und fünfte Lamentation mit identischen Buchstabenund Jerusalem-Sätzen. Ein Schaubild zeigt die Position der identischen Sätze:

Lamentatio III.

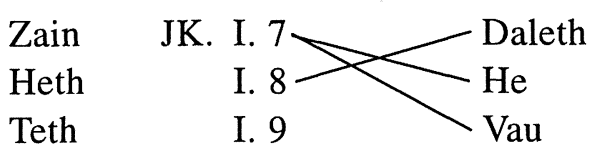

Lamentatio V.
JK. III. 10, 11
III. $13,14,15$
III. 16, 18, 17, 19, 20, 21 .

Teth aus der Lamentatio III ist eine Variante des Zain-Satzes.

Handls Lamentationes IV und VI sind durch gemeinsamen Buchstabensätze und einem Jerusalemrefrain miteinander verzahnt. Noch auffälliger sind Gemeinsamkeiten in Threni-Sätzen, die eine Brücke über eine andere Lektion hinwegschlagen, und so den gesamten Zyklus in hohem Maß musikalisch verzahnen. Als auffälliges Beispiel mag hier der erste Vers aus der Lamentatio IV "Ego vir videns" und der erste Vers aus der Lamentatio VI "Bonus est Dominus" betrachtet werden.

\section{Notenbeispiel 5}

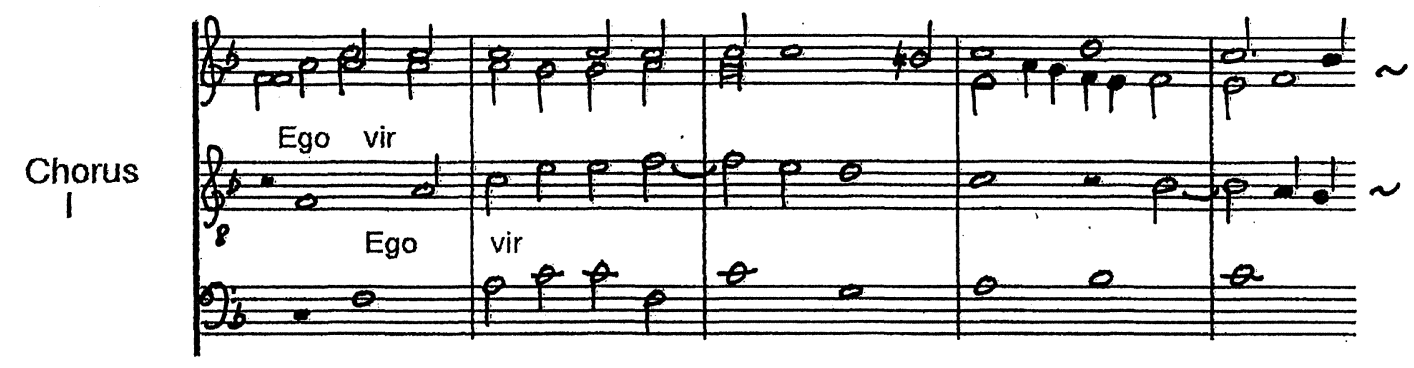




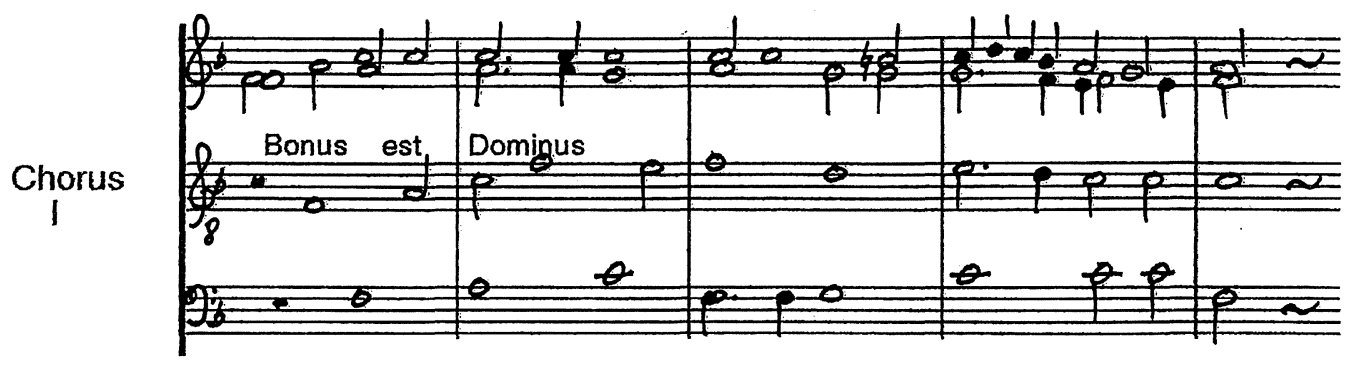

Ein anonymer Lamentationszyklus, ${ }^{33}$ der handschriftlich in einer englischen Bibliothek überliefert wurde, stammt vermutlich aus Norditalien der 2. Hälfte des 16. Jahrhunderts. ${ }^{34}$ Wiederkehrende Sätze wie in den bis jetzt beschriebenen Einzellektionen und Lamentationszyklen sind in dem anonymen Zyklus nicht feststellbar. Doch bringt eine bestimmte kompositorische Idee in ihrer systematischen Anwendung einen gewissen musikalischen Zusammenhalt. "All but two or three of the 25 settings of the words that represent Hebrew letters are built on sequential basses." 35 Bei den sequenzierenden Bässen handelt es sich meist um eine punktierte, sehr kurze Floskel.

Interessant ist, wie in vielen Vokalisen-Sätzen die übrigen Stimmen sich durch Imitationen der kurzen Floskeln am musikalischen Zusammenhalt beteiligen. Besonders gut nachzusehen ist dies in der L.s. des F.q. So startet in der Mensur $353^{36}$ eine punktierte, aufsteigende Floskel, die einmal nach oben sequenziert und danach auf verschiedenen anderen Stufen im Bassus auftritt. Der Diskant setzt als Kontrapunkt eine Gegensequenz. In der Mensur 358 hat der Diskant die Baßfloskel. Im folgenden Vers-Satz "Omnis populus" tritt diese Floskel in der Mensur 374 im Tenor secundus und zweimal in den Mensuren 390-93 im Tenor primus auf. In dem sich anschließenden Jerusalemrefrain erscheint diese Floskel einige Male in originaler und in modifizierter Form in verschiedenen Stimmen, wobei bei unserer Betrachtung nur nahe Varianten berücksichtigt werden und Diminuitionen und Augmentationen dieser Floskel unberücksichtigt bleiben.

Der aufsteigende Pentachord und dessen absteigende Umkehrung und Verlängerung der Tonleiter zeigen sich als prägende Bestandteile dieses Lamentationszyklus. Dafür spricht sein regelmäßiges Auftreten, das nicht den Stellenwert von schlichten Durchgangsnoten hat. Beginnend im Tenor, Mensur 31-33 der L.p. des F.q., tritt diese Figur ungefähr 100 mal, verteilt auf alle 9 Lektionen, auf und verleiht dem Zyklus einen hörbaren, wenn auch musikalisch kleinen Nenner.

33. British Library, Royal Appendix MSS 12-16.

34. Vergl. Bettley, 1992. S. $201 \mathrm{ff}$.

35. Charles W. Warren, The Music Of Royal Appendix 12-16, ML, LI (1970). S. 362.

36. David T. Flanagan, Polyphonic Settings Of The "Lamentations Of Jeremiah" By Sixteenth Century English Composers. (Volumes I and II), Diss. Cornell University, 1990. 
Wie diese Figur über die Sätze hinweggreift, kann man beim Nebeneinander zweier Buchstabensätze gut beobachten, wobei die motivische Verzahnung auch über die tonartlichen Grenzen des 2. Modus hinweggreift.

Notenbeispiel 6 (Caph, Mensur 350 ff. der L.s. des F.q. und des Ain, Mensur 383 der L.t. des F.s.)
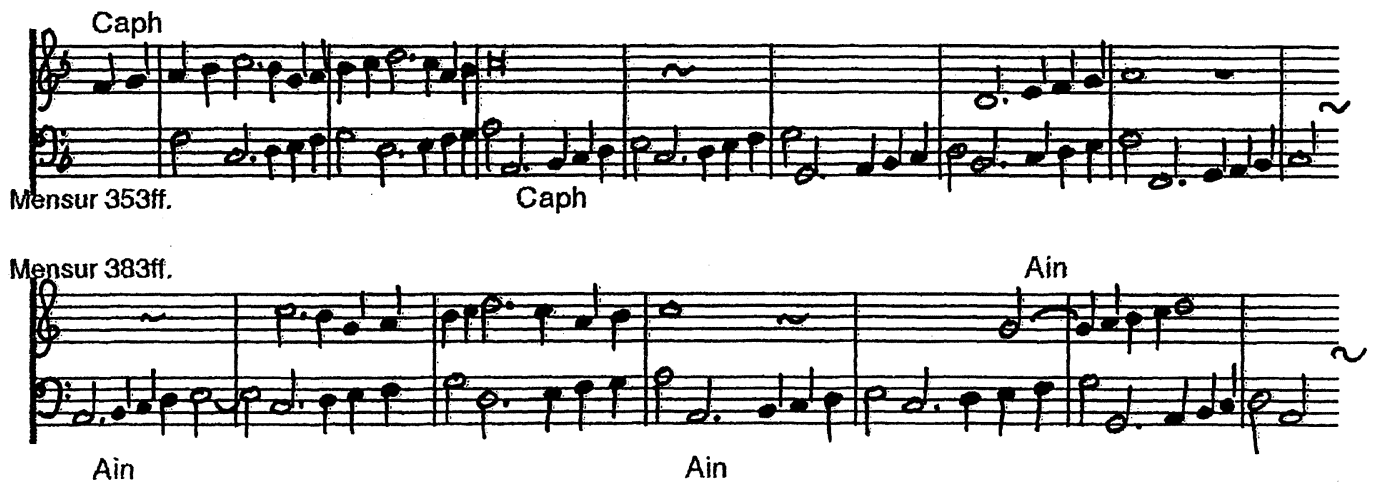

Mit Fabrizio Dentices Lamentationen (Mailand, 1593) liegt ein weiterer musikalischer Zyklus vor, der ein wiederkehrendes Element aufzuweisen hat. Keine der fünf Stimmen wiederholt eines der kurzen Motive mehr als einmal. ${ }^{37}$ Aber eine "marvelous augmented dissonance is found with some frequency throughout the work." 38 Dabei übersieht Schmidt, daß Dentice seine Lamentationen an anderer Stelle viel aufälliger mit einer Regelmäßigkeit versieht. Die 3 Lektionen des feria 6. In Parasceve ${ }^{39}$ sind durch gleiche Jerusalem-Refrains miteinander verzahnt.

Im Manuskript O 31 der Biblioteca Vallicelliana in Rom finden sich die Responsorien und die beiden Lamentationszyklen von Emilio De'Cavalieri, ${ }^{40}$ die 1599 in Pisa und 1600 in Rom zum ersten Mal aufgeführt wurden. In Cavalieris Lamentationen wechseln polyphone und monodische Kompositionsmittel ab. Wiederkehrende Sätze verzahnen sowohl jeden LamentationsZyklus in sich als auch beide Zyklen miteinander.

Das Manuskript mit Cavalieris Lamentationen und Responsorien gehört in die oben aufgeführte Gruppe, die in der Überschrift auf zyklische Zusammenstellung hinweist. ${ }^{41}$ Trotz unterschiedlicher Entstehungszeiten und -orte sind beide Lamentationszyklen miteinander musikalisch

37. Vergl. Carl Brandon Schmidt, Fabricio Dentice And His Polyphonic Lamentations Of 1593, unveröff. M.A.Arbeit, Harvard University, Cambridge, Massachusetts, 1965. S. 33.

38. Ebda., S. 26.

39. Siehe die Edition in Schmidt, 1965. S. $53 \mathrm{ff}$

40. Cavalieri. S. XIII.

41. Siehe Faksimile in: Cavalieri. S. LXVII. 
verknüpft, indem Cavalieri die erste und letzte der 9 Lektionen von 1600 mit dem gleichen fünfstimmigen Jerusalemrefrain versieht. In den Lamentationen von 1599 setzt Cavalieri identische Jerusalemrefrains ein, auf die er in den Lamentationen von 1600 zurückgreift. Im Falle der Lektionen mit Moduswechsel besteht die Möglichkeit eines Alternativsatzes, auf den im Zyklus von 1600 ebenfalls zurückgegriffen wird.

Giovanni Francesco Capellos Lamentationen (Verona, 1612), deren Drucktitel ... Da cantarsi il Mercodi, Giovedi, e Venerdi/Santo di sera à Matutino ... auf einen vollständigen liturgischen Zyklus hinweist, zeigen gewisse strukturelle Gemeinsamkeiten mit Cavalieris Lamentetionszyklen - Buchstabensätze und Jerusalemrefrains sind dem fünfstimmigen (bzw. sechstimmigen) Chor vorbehalten. Capellos Lektionen "are framed by largely chordal sections in the Venetian style for the full ensemble, but the Lamentations themselves are entrusted to soloists ..."42 Dieser systematische Wechsel von solistischen Verssätzen (die mitunter auch vom Chor aufgegriffen werden,) und chorischen Buchstaben- und Jerusalemsätzen kann als Plan für eine Zyklusgestaltung aufgefaßt werden, doch soll die Dispositionsthematik hier nicht weiter vertieft werden. Eine besonders auffällige Wiederkehr musikalischen Materials begegnet uns in Capellos L.t. vom Karfreitag, “ ... utilizing a tenor solist and an instrumental ritornello. The rubric, first four verses and refrain ... are all sung to six slightly modified repetitions of the same arioso melody. Each repetition is separated from the others by a triple-meter ritornello, which is also repeated at the conclusion." ${ }^{43}$ Wiederkehrende instrumentale Sätze, zumindest in einer Lektion, geben Capellos Lamentationszyklus im frühen 17. Jahrhundert eine neue Qualität. Capellos und Cavalieris instrumentale und monodische Bestrebungen in Verbindung mit wiederkehrenden musikalischen Merkmalen wurden nicht gleich von anderen Komponisten aufgegriffen. Zunächst wurden sie in der ersten Hälfte des 17. Jahrhunderts polyphon gesetzt, man denke besonders an die spanischen und portugiesischen Meister, von denen am Anfang E. de Brito erwähnt wurde. Dazu findet sich am Ende der Stimmbücher des op. XXII (1609) von Lodovico da Viadana eine Bemerkung: "non si é fatto il basso continuo a queste Lamentationi, perché a dirne il vero questa sorte di Musica che recita, farà sempre miglior effetto solo [con] quattro buone voci, che cantano con gravità, e schietto, [piuttosto] che esser accompagnata con istrumenti." 44 Viadanas Lamentationen sind mit ihren wiederkehrenden Jerusalemsätzen ein weiteres Beispiel für zyklische musikalische Gestaltung. Diese und andere Lamentationszyklen des frühen 17. Jahrhunderts zeigen durch ihre Bindung an die Liturgien der Kathedral- und Klosterkirchen ein konservatives Element, das sich im (polyphonen) a capella Stil widerspiegelt.

42. Jeffrey Kurtzman, Giovanni Francesco Capello, An Avant-Gardist Of The Early Seventeenth Century, MD, Vol. XXXI (1977), S. 162.

43. Ebda.

44. Zitiert aus Giovanni Acciai \& Lucio Christante, Booklet zu Lodovico da Viadana: Responsoria et Lamentationes, CD stradivarius 33444,1996, S. 7. 


\section{Fazit}

Die oben aufgeführte Auswahl zeigt, wie wiederkehrende Sätze oder Satzvarianten und andere Merkmale mehrstimmige Lamentationen zu musikalischen Zyklen formen. Diese Lamentationszyklen treten über den gesamten, oben beschriebenen Zeitraum mit dem regionalen Schwerpunkt Norditalien auf. Der regionale Schwerpunkt kann mit der norditalienischen Konzentration des Druckmarktes, besonders in Venedig, erklärt werden. Die exemplarische Untersuchung zeigt, daß die zyklische Gestaltung nicht die ganze Gattung der polyphonen Lamentationen abdeckt, dennoch kann sie als gattungsspezifisch angesehen werden, da sie sich als Gestaltungsmittel einzelner Komponisten zeigt, die bei der Vertonung einer umfangreichen liturgischen Textvorlage vermutlich eine gewisse musikalische Geschlossenheit erreichen wollten. Das Nebeneinander verschiedener Lamentations-Zyklen zeigt Spielarten wiederkehrender Satzpartikel und Sätze, die über die Klauselsystematik, die konsequent strophische Anlage und das wörtliche Wiederholen bestimmter Sätze bis hin zur Wiederkehr paraphrasierter Satzmodelle reicht. Zyklische Anlagen findet man sowohl in den polyphonen, den choralgebunden und motettischen, als auch in den monodischen Lamentationen. 